12 Lajoie, K. R., thesis, Univ. California, Berkeley (1968)

13 McCarthy, R. D., and Duthie, A. H., J. Lipid Res., 3, 117 (1962).

${ }^{14}$ Reitz, R. C., and Hamilton, J. G., Biochem. Physiol., 25, 401 (1968).

15 de Souza, N. J., and Nes, W. R., Science, 162, 363 (1968).

16 Ikekawa, N., Morisaki, N., Tsuda, K., and Yoshida, T., Steroids, 12, 41 (1968).

\section{Nature of Quickclays}

THE strength of clay soils in situ is often greater than the disturbed or remoulded strength and the ratio of these two values is a measure of the "sensitivity" of the soil; this may vary from $\sim 1$ for a London clay to $\sim 1,500$ for a Canadian Leda clay ${ }^{1}$. The extremely sensitive soils, known as quickclays, are chiefly found in northern North America and Scandinavia, and they present a considerable geotechnical hazard because of a tendency to failure by landslide. The loss of strength at failure is remarkable--the material changes from a strong brittle soil into a viscous liquid, and this has proved difficult to explain.

The only substantial current theory is that of Rosenqvist ${ }^{2}$ who proposed that the typical quickclay properties were produced by postdepositional leaching. This theory requires that the quickclay structure be formed by clay mineral particles in a "cardhouse" structure and that the cohesion in this system be reduced by the removal of soil water ions by fresh water leaching, the initial deposition being in sea water. The theory has been criticized ${ }^{3}$ and does not explain certain observations on Canadian quickclays ${ }^{4}$.

Rosenqvist, and most other investigators, have considered quickclays to be real clay soils and have expected the clay mineral content to determine the failure mechanism. Analyses of landslide soils ${ }^{5,6}$ show that clay minerals are not present in significant amounts and suggest that the other soil particles plav the key role in determining quickclay properties. If a supply of fine non-clay mineral soil material is a requirement for quickclay formation then the geographical distribution is explicable. The abundant fine material formed by glacial action is incorporated into the quickclays.

There are essentially two types of interparticle bond in soils, long range active bonds and short range inactive bonds. The long range bonds exist between clay particles and depend on the charge of the particles, the presence of solute soil water ions and the polarized nature of water. The short range bonds exist between uncharged particles (for example, quartz silt particles) and depend on particle contact; once the contact is disturbed the bond is ineffective. The most essential requirement for quickclay formation is that short range bonds predominate in the soil system.

Leaching an ion rich soil weakens the clay-clay bonds and thereby effectively increases the proportion of short range bonds. Thus leaching can be seen as one factor contributing to quickness, but not the key factor. In fact a soil composed completely of clay minerals cannot be leached into a state of quickness ${ }^{3}$. The soil particles must also have a very low settling velocity in water and the system must have a high water content; this allows the transition from solid to liquid at failure to be accomplished.

Normal engineering soil classifications are based entirely on size and this has obscured the contribution made to soil properties by particles of, or approaching, clay size but lacking clay mineral properties. The relationship of the various soil particles to each other is shown in Fig. 1 in which the bondweight ratio $R$ is plotted against particle size. The nature of $R$ has been defined and discussed elsewhere ${ }^{7}$; it is the ratio of the cohesive force between particles to particle size. When $R=1$ the forces effectively balance; when $R<1$ the particle weight obscures the effect of the interparticle forces and the soil is termed cohesionless but when $R>1$ the system is cohesive and the particle interaction has more effect on the properties

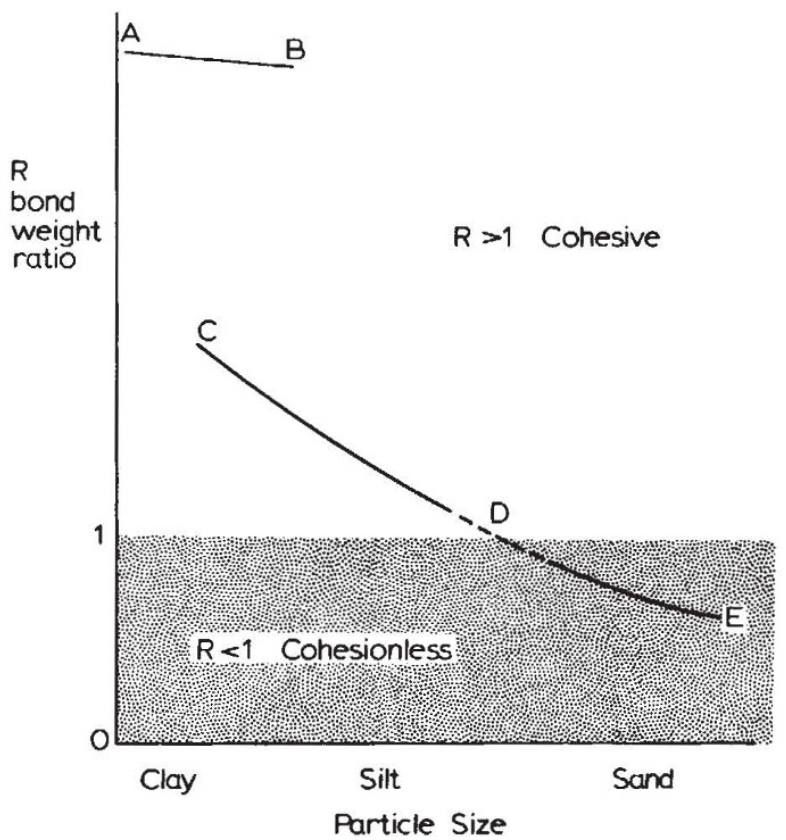

Fig. 1 Relationship of bond-weight ratio $R$ to particle size for soil particles. $A B$, clay particles; $C D E$, non-clay particles (usually quartz in real soils).

than the weight of the particles. The line $A B$ represents the clays with large $R$ values; line $C D E$ represents the non-clay mineral particles ranging from clay size to sand size. There is a certain lack of particles in the $D$ region because of particle formation factors which result in the predominant quartz particles having a bimodal distribution ${ }^{8}$. Quickclays chiefly contain particles from around the $C$ region and these, with short range bonds operating, determine the quickclay properties.

Three basic types of soil can be distinguished using criteria of particle size and interparticle bond type: (1) small particles, long range bonds (the real clays and clay soils); (2) small particles, short range bonds, and (3) larger particles, short range bonds (the sands). Previous investigators have tended to assume that quickclays were type 1 soils, with perhaps a slight tendency to "high silt content" but it is more satisfactory to consider them as type 2 soils. In mineralogical terms, however, quickclays are not really clays at all.

\section{J. Smalley}

Department of Civil Engineering,

University of Leeds, Leeds LS2 9JT

Received March 8; revised April 28, 1971.

1 Penner, E., Nature, 197, 347 (1963).

2 Rosenqvist, I. Th., Geotechnique, 3, 195 (1953).

3 Pusch, R., and Arnold, M., Eng. Geol., 3, 135 (1969).

4 Penner, E., Canad. J. Earth Sci., 2, 425 (1965).

5 Peck, R. B., Ireland, H. O., and Fry, T. S., U. Illinois Cir. Eng. Dept. Soil Mech. Series, No. 1 (1951).

6 Beland, J., Proc. Geol. Assoc. Canada, 8, 143 (1956).

7 Smalley, I. J., J. Soil Sci., 21, 154 (1970).

8 Vita-Finzi, C., and Smalley, I. J., J. Sediment. Petrol., 40, 1367 (1970).

\section{X-ray Diffraction Evidence for Crystalline Order and Isotropic Compression during the Shock-wave Process}

WE have reported ${ }^{1}$ observing $\mathrm{X}$-rays diffracted from material which was undergoing shock-wave compression. The shock wave was generated by a conventional high-explosive, planewave generator. The four-channel scintillation detector used 